\title{
The Impact of Hindlimb Suspension on the Rat Eye: A Molecular and Histological Analysis of the Retina
}

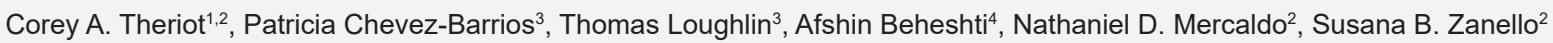

${ }^{1}$ University of Texas Medical Branch, Galveston, TX; ${ }^{2} K B R$, NASA Johnson Space Center, Houston, TX; ${ }^{3}$ The Houston Methodist Hospital and Houston Methodist Academic Institute, Houston, TX; ${ }^{4} \mathrm{KBR}$, NASA Ames Research Center, Moffett Field, CA

Abstract

The Spaceflight Associated Neuro-ocular Syndrome (SANS) is hypothesized to be associated with microgravity-induced fluid shifts. There is a need for an animal model of SANS to investigate its pathophysiology. We used the rat hindlimb suspension (HS) model to examine the relationship between the assumed cephalad fluid shifts, intraocular (IOP) pressure and the molecular responses in the retina to the prolonged change in body posture. Long evans rats were subjected to HS up to 90 days. Animals completing 90-day suspension were further studied for recovery periods up to 90 additional days in normal posture. With respect to baseline, the average IOP increase in HS animals and the rate of change varied by cohort. Transcriptomics evidence supported a response to HS in the rat retina that was affected by age and sex. Several molecular networks suggested stress imposed by HS affected the retinal vasculature, oxidative and inflammation status, pigmented epithelium and glia. The CSNK1A1-TP53 pathway was implicated in the response in all cohorts. Sex-specific genes were involved in cytoprotection and may explain sex-dependent vulnerabilities to certain eye diseases. These results support the hypothesis that changes in the biology of the retina subjected to simulated microgravity involve both the neural and vascular retina.

Keywords

Spaceflight associated neuro-ocular syndrome $\bullet$ retina $\bullet$ microgravity $\bullet$ hindlimb suspension $\bullet$ transcriptomics $\bullet$ histology

\section{INTRODUCTION}

The Spaceflight Associated Neuro-ocular Syndrome (SANS) is a condition manifested in astronauts during long-duration space missions on the International Space Station (Mader et al., 2011, Lee et al., 2017, Lee et al., 2018). It is hypothesized that the exposure to microgravity, and the associated fluid shifts occurring in consequence, are involved in its etiology (Zhang and Hargens 2018, Huang et al., 2019a, Mader et al., 2019, Wostyn et al., 2020). The condition is characterized by eye globe flattening and hyperopic shift, optic nerve sheath distension, choroidal folds and optic disc edema (Mader et al., 2011, Laurie et al., 2019, Stenger et al., 2019, Lee et al., 2020, Wojcik et al., 2020). Additionally, other signs have occasionally been detected, like cotton wool spots and scotoma. Thanks to newer optical computer tomography (OCT) technology, optic disc edema has been shown to be more prevalent than previously believed and occurring in both in males and females (Mader et al., 2013, Lee 2018, Lee et al., 2018). Further research is needed to test whether fluid shift and/or an increase in intracranial pressure (ICP) are mediating factors leading to these symptoms (Lee et al., 2016).
Recent investigations have reported an association of SANS with 1-carbon metabolism status and genetic determinants (Zwart et al., 2017). Astronauts manifesting ophthalmic issues after flight had higher plasma concentrations of metabolites associated with the 1-carbon metabolic pathway (Zwart et al., 2012). Single nucleotide polymorphisms were linked to two enzymes (methionine synthase reductase, MTRR A66G, and serine hydroxymethyltransferase-1, SHMT1 C1420T) in astronauts described with SANS as well as in subjects confined to strict 6 degree head-down tilt bed rest in whom signs of SANS were replicated (Zwart et al., 2016, Zwart et al., 2019). Previous studies in subjects maintained in head-down tilt bed rest had not shown clinical SANS signs, however statistically significant thickening of the retinal nerve fiber layer had been observed (Taibbi et al., 2016).

While clinical and subclinical symptoms have been identified in the astronaut cohort, the presence of changes at the cellular and molecular level should not be discarded. Early alterations in retinal biology may lead to retinal dysfunction at later stages and this possibility should be investigated. This would be similar to what occurs in several clinical ophthalmic conditions, where inflammation and oxidative stress gradually

\footnotetext{
†Corresponding author: Susana Zanello
}

E-mail: susana.b.zanello@nasa.gov 
affecting the neuronal, glial and vascular retinal homeostasis (Moran et al., 2016, Almasieh and Levin 2017, Tamm et al., 2017, Rubsam et al., 2018, Selvam et al., 2018) are implicated in the pathology.

The pathophysiology of SANS is not understood and an examination of the retina in affected eyes is needed to better characterize the risk. While OCT allows a quasi-histologic analysis of the retina (i.e. retina fiber layer thickening evidencing edema), a deeper histologic and molecular analysis is only possible using animal models. One of the objectives of the present study was to evaluate hindlimb suspension (HS) in rodents as an analog for the study of SANS.

This work also investigates the sex and age differences in the response to HS in rats. The rodent HS ground analog model involves suspending a rat or mouse by the tail adjusted to approximately 30 degrees head-down tilt. Hindlimb suspension, a widely used model of microgravity deconditioning (Morey-Holton and Globus 2002, Morey-Holton et al., 2005, Globus and Morey-Holton 2016, Tahimic et al., 2019), was used in this study to examine changes elicited in the retina of rats in order to assess its value as a model of SANS. This was performed under the assumption that cephalad fluid shift in a rodent (quadruped) is akin to that occurring for humans in microgravity (bipeds on the ground) (Musacchia and Fagette 1997, Morey-Holton et al., 2005). Limited characterization of the effects of HS on the eye has been done to date (Mao et al., 2019), and to our knowledge, none focused on the gene expression in the retina. Sex-dependent gene expression independent of treatment is here reported for the rat retina. However, a common pathway involving key regulator genes in the CSNK1A1 and TP53 network seem to be common to all cohorts examined. A differential response to HS under the influence of the estrogenic environment in these animals may lead to a different susceptibility to SANS in females versus males. The results presented here support the hypothesis posed that behind the clinically visible signs of SANS there are underlying changes in the biology of the retina subjected to simulated microgravity, which may involve both the neural and vascular retina.

\section{MATERIALS AND METHODS}

\section{Animals}

This workwas performed underatissuesharing agreementwith Dr. Charles Fuller (Department of Neurobiology, Physiology and Behavior, University of California, Davis). The study was conducted with approval from the UC Davis and NASA Johnson Space Center Institutional Animal Care and Use Committees. Long Evans rats were purchased from Taconic (Albany, NY). The study encompassed three cohorts: young males (YM): 3 months-old at the start of the experiment, young females (YF): 3 months-old at the start of the experiment, and old males (OM): 9 months-old at the start of the experiment.

The experimental protocol used the HS method described by Morey-Holton (Morey-Holton et al., 2005), where the rats were held in hindlimb suspension by the tail for $7,14,28,45$ and 90 days. Subgroups of the 90 -day HS rats were studied for recovery periods of $7,14,28,45$ or 90 days, during which they were returned to the weight bearing position. Maximum length of the entire animal protocol was 180 days. All HS animals had corresponding age-matched cage controls. All animals had ad libitum access to standard chow food and water and were subjected to light/dark (LD) cycles of 12:12 hr LD. After completion of the experiment, rats were euthanized under anesthesia at the specific time points. Tissue was harvested and preserved in solution, then shipped from UC Davis to Johnson Space Center for preparation and analysis as described in further detail below. Complexity, length and animal attrition of the experimental scheme resulted in an incomplete sample record for some time points within each cohort, as noted in the results.

\section{Intraocular pressure (IOP)}

Intraocular pressure (IOP) was measured by tonometry using a rebound tonometer (Icare $\AA$ TONOLAB, Vantaa, Finland) according to manufacturer specifications. The instrument was applied to the cornea of both the left and right eye to collect IOP at baseline, during the 90-day HS period, and during the 90-day normal posture recovery period. The average of 6 consecutive readings was used as the IOP measure for each data sample.

\section{IOP temporal and demographic analysis}

Linear mixed-effects models were constructed to quantify temporal changes in IOP by sex (male, female) and age (young, old) cohorts. Separate regression models were constructed in which IOP was regressed onto each demographic factor (e.g., sex), time and their interaction, and side (left, right). Due to the small sample size, only a random-intercept model was used to account for data dependencies due to multiple measurements. For each model, main effect estimates were summarized (e.g., average IOP among males at time 0 , at time 7 , etc.) along with their $95 \%$ confidence intervals. Next, linear combinations of parameter estimates were estimated to summarize temporal effects at pre-specified time points (during treatment and during the recovery phase) and differences in rates of change by cohort. Both unadjusted and FDR-adjusted $p$-values were generated for all comparisons. All analyses were performed using R 3.6.1 [release date 7/5/2019]. 


\section{Histology and Immunohistochemistry}

The left eyes from the rats were enucleated and immediately immersed in Excalibur Pathology's proprietary fixative (Excalibur Pathology, Inc, Norman, OK) at room temperature until being paraffin embedded and sliced in $5 \mu \mathrm{m}$ thick sections. Non-consecutive eye sections (separated by 50$150 \mu \mathrm{m})$ were stained with standard hematoxylin-eosin (H\&E) procedures and morphologic changes reviewed (3 levels/steps of the eye) on an anterior posterior plane to review the pupil-optic nerve plane per eye after evaluation of completeness of structures and representation of different ocular planes. Immunostaining of the retina and optic nerve was performed using glial fibrillary protein (GFAP) and vimentin antibodies. The slides were deparaffinized, rinsed with deionized water, and placed in Dako 1X Wash Buffer. All reagents were prepared according to manufacturer's instructions, and incubations were performed in a humid chamber at room temperature. Antigens were unmasked with Dako Target Retrieval Solution at $99^{\circ} \mathrm{C}$. Peroxidase was blocked with $3 \% \mathrm{H}_{2} \mathrm{O}_{2}$, and biotin was blocked with Vector Avidin/Biotin Blocking Kit (Vector Laboratories, Burlingame, CA). Dako Serum Free Protein Block was used to prevent non-specific protein binding. Slides were incubated for 30 minutes in ready-to-use mouse antibody to rat GFAP (Dako, Carpinteria, CA) for 30 minutes. Slides were washed with buffer and incubated for 30 minutes with biotinylated rat antimouse IgG (Vector Laboratories, Burlingame, CA) diluted 1:100 in antibody diluent. After washing in buffer, slides were incubated in phosphatase-labeled streptavidin (Seracare $\mathrm{KPL}$, Milford, MA) for 30 minutes followed by incubation in wash buffer for 5 minutes. Slides were then incubated for 3 minutes in Dako Liquid Permanent Red chromogen, prepared with $25 \mu \mathrm{L}$ of Liquid Permanent Red chromogen per $3 \mathrm{~mL}$ of Liquid Permanent Red Substrate Buffer, or with $\mathrm{DAB}$ chromogen. Slides were placed in deionized water, washed in buffer, counterstained with Hematoxylin 2 (ThermoFisher Scientific, Waltham, MA), and coverslipped. Single immunostain for Müller cells and glial cells using rabbit polyclonal antibody to vimentin (Abcam, Cambridge MA) diluted 1:1500 in antibody diluent Dako DAB+ Substrate Chromogen System and conventional automated staining was performed. Negative (no primary antibody) and positive controls (rat retina) were performed every time slides were immunostained.

\section{Histopathology}

The eyes were reviewed by an ophthalmic pathologist masked for type of animal (control vs HS, female vs male). Findings of ocular features, in particular the retina and optic nerve, were recorded and graded. The grading for any lesion was as follows: $0=$ no lesion found; $1=$ faint and minimal findings present in less than a quarter of the tissue; 2 = obvious findings seen in more than a quarter to a half of the tissue examined; $3=$ more than half to two thirds of tissue examined and intense; $4=$ intense and diffuse change present. The findings were recorded for cell type in each tissue type and exact location in the eye.

\section{RNA processing}

The right eyes from rats were enucleated and immediately submerged in RNAlater (ThermoFisher Scientific, Waltham, MA). Retinas were removed from the posterior eye cup and total RNA was isolated from the retina using the AllPrep DNA/RNA Micro kit (Qiagen, Valencia, CA). RNA quality was determined using an Agilent 2100 Bioanalyzer (Agilent Technologies, Santa Clara, CA) and RNA concentration was determined using a NanoDrop 2000 (ThermoFisher Scientific, Waltham, MA). Microarray analysis services were provided by the Baylor College of Medicine Genomic and RNA Profiling Core facility (Houston, TX). Affymetrix GeneChip $®$ Rat Genome 2302.0 microarrays were used with a sample size of $n=4$ per experimental group. No microarrays were run if samples were missing in an experimental group (for example, no microarray was performed for YM 180 days, since only $n=2$ eyes were available).

\section{Transcriptomic Analysis}

Two transcriptomic analysis approaches were used in order to increase confidence in the results and to exploit unique features of each bioinformatics pipeline. In the first one, Qlucore Omics Explorer v 3.5 (Qlucore, Lund, Sweden) was used to generate Principal Component Analysis (PCA) plots. CEL files were normalized using the RMA method and intensity values of each probe set were $\log _{2}$ transformed. PCA plots were produced with multigroup comparison ANOVA, filtering for variance. Two-group comparisons were performed using t-tests between selected experimental conditions and time points as applicable.

In the second method, raw data was first background adjusted and quantile normalized using RMAExpress (Bolstad et al., 2003). Normalized data was also imported into MultiExperiment Viewer (Saeed et al., 2006) and statistically significant genes were determined by ANOVA with an adjusted $p$-value $<0.05$ for all comparisons to take forward for pathway analysis. Next, pathway analysis of the selected genes was performed by using a fold-change $\geq 1.2$ (or $\leq-1.2$ ) comparing experimental conditions versus controls. This fold-change is an arbitrary value which, in our experience, produces the maximum amount of the genes above the noise. One method for observing pathway relationships was done using IPA software (Ingenuity Systems, Qiagen, Valencia, CA). Upstream regulator analysis from IPA identified any molecule that affected the expression or function of the measured downstream target genes. The activation state 
Table 1. Average IOP values by sex, side, and time.

\begin{tabular}{|c|c|c|c|c|c|c|}
\hline \multirow[t]{2}{*}{$\begin{array}{l}\text { Time } \\
\text { (days) }\end{array}$} & \multicolumn{2}{|c|}{ YM Cohort } & \multicolumn{2}{|c|}{ YF Cohort } & \multicolumn{2}{|c|}{$\begin{array}{l}\text { Cross-sectional Sex Comparisons } \\
\text { (Female-Male) }\end{array}$} \\
\hline & Left Eye $(95 \% \mathrm{Cl})$ & Right Eye $(95 \% \mathrm{Cl})$ & Left Eye $(95 \% \mathrm{Cl})$ & Right Eye $(95 \% \mathrm{Cl})$ & Difference $(95 \% \mathrm{Cl})$ & $p$-value (p-FDR) \\
\hline 0 & $17.9(16.9,18.9)$ & $18.1(17.1,19.1)$ & $19.1(18.2,19.9)$ & $19.3(18.4,20.1)$ & $1.15(-0.08,2.39)$ & $0.067(0.091)$ \\
\hline 7 & $19.4(18.5,20.3)$ & $19.6(18.7,20.5)$ & $19.7(18.9,20.5)$ & $19.9(19.1,20.7)$ & $0.31(-0.80,1.43)$ & $0.582(0.644)$ \\
\hline 14 & $20.8(19.9,21.7)$ & $21.0(20.1,21.9)$ & $20.3(19.5,21.2)$ & $20.5(19.7,21.4)$ & $-0.48(-1.65,0.68)$ & $0.411(0.486)$ \\
\hline 28 & $23.2(22.0,24.3)$ & $23.4(22.2,24.5)$ & $21.4(20.4,22.5)$ & $21.7(20.6,22.7)$ & $-1.73(-3.24,-0.22)$ & $0.025(0.036)$ \\
\hline 45 & $24.5(23.2,25.9)$ & $24.7(23.4,26.1)$ & $22.4(21.2,23.6)$ & $22.6(21.4,23.8)$ & $-2.16(-3.90,-0.42)$ & $0.015(0.023)$ \\
\hline 90 & $21.1(20.1,22.1)$ & $21.3(20.3,22.3)$ & $22.6(21.7,23.5)$ & $22.8(21.9,23.7)$ & $1.51(0.18,2.83)$ & $0.026(0.037)$ \\
\hline 97 & $20.8(19.8,21.9)$ & $21.1(20.0,22.1)$ & $22.6(21.7,23.6)$ & $22.8(21.9,23.8)$ & $1.77(0.42,3.13)$ & $0.011(0.016)$ \\
\hline 104 & $20.8(19.8,21.9)$ & $21.1(20.0,22.1)$ & $22.7(21.7,23.6)$ & $22.9(21.9,23.9)$ & $1.84(0.46,3.21)$ & $0.009(0.014)$ \\
\hline 180 & $26.2(23.9,28.4)$ & $26.4(24.1,28.7)$ & $24.2(21.8,26.7)$ & $24.4(22.0,26.9)$ & $-1.94(-5.27,1.40)$ & $0.254(0.307)$ \\
\hline
\end{tabular}

Baseline and hindlimb suspended (HS)-induced IOP changes by sex group (male, female), side (left, right) and time (0-180). All estimates are based on a linear-mixed effects model (assuming the random-effect $=0$ ). For example, the average IOP within the young male cohort at baseline (day 0) was $17.9 \mathrm{mmHg}(95 \% \mathrm{Cl}: 16.9-18.9)$ [left eye]. The similar value within the young female cohort was 19.1 (18.2 - 19.9). The difference (female-male) in these average values was 1.15 (-0.08- 2.39). Given side was modeled as a main-effect only, the cross-sectional sex differences are the same for the left and right eye (e.g., 19.1-17.9=1.2 [day 0; left], 19.3-18.1=1.2 [day 0; right]; slight differences due to rounding).

of each upstream regulator from the experimental data set was determined by calculating the $z$-score $(\geq 2$, activated or $\leq-2$, inhibited). Gene set enrichment analysis (GSEA) was performed using the entire list of genes and with leading edge analysis as previously described by Subramanian et al (Subramanian et al., 2005). Significant gene sets between age groups were considered with false discovery rate (FDR) $\leq$ 0.05 . An unbiased method to identify key genes/drivers were determined as previously reported in Beheshti et al. (Beheshti et al., 2015) by locating the overlapping genes involved in predicting significant upstream regulators, biofunctions, and GSEA gene sets (which includes the following gene sets: C2, C5, and hallmarks gene set). More specifically, for each set of genes under analysis, the association with statistically significant pathways and functions were determined through both IPA and GSEA. Common genes were determined to be involved by an analysis of both IPA upstream regulators and biofunctions outputs. These sets of genes were further compared to the GSEA's leading edge genes (FDR $\leq 0.05$ ). The overlapping genes between these two analyses were considered to be the key genes involved and in control of the majority of predicted functions and activity with the system being analyzed. In previous publications, we have validated key genes determined with this method through experimental approaches involving Western blots, qPCR, and other functional methods (Beheshti et al., 2015, Ravi et al., 2016). These experiments proved that the key genes determined with this bioinformatics interrogation method were indeed involved in the system being studied.

\section{RESULTS}

\section{Intraocular pressure increases uniquely according to sex and age during $\mathrm{HS}$}

Model-based average IOP estimates, along with crosssectional and temporal comparisons, are summarized in Tables I-IV. In Tables I and III, results are presented by side (left - oculus sinister [OS] and right - oculus dexter [OD]) even though there was insufficient evidence to conclude that a side-effect was present in these data. More specifically, the estimate associated with side in the analysis of young male/ female cohorts was $0.21(95 \% \mathrm{Cl}$ : $-0.40-0.82 ; \mathrm{p}=0.504)$ and was $0.11(-0.64-0.86)$ when analysing the young/old male cohorts.

From Table I, the average OS IOP at baseline was 17.9 (16.9-18.9) and 19.1 (18.2 -19.9) in the YM and YF cohorts, respectively. This corresponds to an average baseline difference (YF-YM) of $1.15(-0.08,2.39)$. By day 45 , the average HS-induced OS IOP estimates increased to 24.5 $(23.2,25.9)$ and $22.4(21.2-23.6)$ which corresponded to an average OS IOP difference of $-2.16(-3.90,-0.42)[p=0.015$, $\mathrm{p}$-fdr=0.023]. Among the YM cohort, the temporal change between day 45 and baseline was $6.62(5.15-8.10 ; p<0.001)$ [Table II]. Similarly, among the YF cohort, this increase was 3.31 (1.97-4.65). Note, temporal differences are the same regardless of side due to the inclusion of only a main-effect of side in the regression model. The differences in these rates of change was $-3.31(-5.31,-1.32)[p=0.001, p-f d r=0.002]$ which 
Table 2. HS-induced IOP temporal changes by sex cohort.

\begin{tabular}{ccccccc}
\hline $\begin{array}{c}\text { Temporal } \\
\begin{array}{c}\text { Comparison } \\
\text { (days) }\end{array}\end{array}$ & \multicolumn{2}{c}{ YM Cohort } & \multicolumn{2}{c}{ YF Cohort } & \multicolumn{2}{c}{$\begin{array}{c}\text { Change Comparisons by Sex Group } \\
\text { (Female-Male) }\end{array}$} \\
\cline { 2 - 7 } & Estimate $(\mathbf{9 5} \% \mathrm{Cl})$ & p-value (p-FDR) & Estimate $(\mathbf{9 5 \%}$ Cl) & p-value (p-FDR) & Difference (95\% Cl) & p-value (p-FDR) \\
\hline $\mathbf{7}$ vs. $\mathbf{0}$ & $1.49(1.15,1.83)$ & $<0.001(<0.001)$ & $0.64(0.34,0.95)$ & $<0.001(<0.001)$ & $-0.84(-1.30,-0.39)$ & $<0.001(0.001)$ \\
$\mathbf{1 4}$ vs. $\mathbf{0}$ & $2.91(2.25,3.57)$ & $<0.001(<0.001)$ & $1.27(0.67,1.87)$ & $<0.001(<0.001)$ & $-1.64(-2.53,-0.75)$ & $<0.001(0.001)$ \\
$\mathbf{2 8}$ vs. $\mathbf{0}$ & $5.27(4.08,6.46)$ & $<0.001(<0.001)$ & $2.39(1.31,3.47)$ & $<0.001(<0.001)$ & $-2.89(-4.49,-1.28)$ & $<0.001(0.001)$ \\
$\mathbf{4 5}$ vs. $\mathbf{0}$ & $6.62(5.15,8.10)$ & $<0.001(<0.001)$ & $3.31(1.97,4.65)$ & $<0.001(<0.001)$ & $-3.31(-5.31,-1.32)$ & $0.001(0.002)$ \\
$\mathbf{9 0}$ vs. $\mathbf{0}$ & $3.19(2.03,4.34)$ & $<0.001(<0.001)$ & $3.54(2.51,4.57)$ & $<0.001(<0.001)$ & $0.35(-1.20,1.90)$ & $0.656(0.700)$ \\
$\mathbf{9 7}$ vs. $\mathbf{9 0}$ & $-3.68(-5.15,-2.22)$ & $<0.001(<0.001)$ & $0.25(-1.09,1.59)$ & $0.714(0.742)$ & $3.93(1.95,5.92)$ & $<0.001(<0.001)$ \\
$\mathbf{1 0 4}$ vs. $\mathbf{9 0}$ & $-3.68(-5.21,-2.16)$ & $<0.001(<0.001)$ & $0.31(-1.08,1.71)$ & $0.659(0.700)$ & $4.00(1.93,6.07)$ & $<0.001(<0.001)$ \\
$\mathbf{1 8 0}$ vs. $\mathbf{9 0}$ & $1.64(-0.76,4.04)$ & $0.180(0.223)$ & $1.86(-0.70,4.42)$ & $0.155(0.201)$ & $0.22(-3.29,3.73)$ & $0.902(0.902)$ \\
\hline
\end{tabular}

Hindlimb suspended (HS)-induced IOP changes compared to baseline (time - 0 days) or during the normal posture recovery period compared to the end of HS (time - 90 days) measurement as well as rates of change comparison by sex cohort (female-male). All estimates are based on a linear-mixed effects model (assuming the random-effect $=0$ ). For example, the average IOP change within the young male cohort between day 7 and day 0 was $1.49 \mathrm{mmHg}(95 \% \mathrm{Cl}: 1.15-1.83)$ after adjusting for side (left/right). The analogous value within the young female cohort was $0.64(0.34-0.95)$. The difference (female-male) in these rates of change was $-0.84(-1.30--0.39)$.

Table 3. Average IOP values by age, side, and time.

\begin{tabular}{ccccccc}
\hline $\begin{array}{c}\text { Time } \\
\text { (days) }\end{array}$ & \multicolumn{2}{c}{ YM Cohort } & \multicolumn{2}{c}{ OM Cohort } & \multicolumn{2}{c}{$\begin{array}{c}\text { Cross-sectional Age Comparisons } \\
\text { (Old-Young) }\end{array}$} \\
\cline { 2 - 7 } & Left Eye (95\% Cl) & Right Eye (95\% Cl) & Left Eye (95\% Cl) & Right Eye (95\% Cl) & Difference (95\% Cl) & p-value (p-FDR) \\
\hline $\mathbf{0}$ & $18.0(16.9,19.0)$ & $18.1(17.0,19.1)$ & $19.1(17.7,20.6)$ & $19.3(17.8,20.7)$ & $1.19(-0.51,2.89)$ & $0.168(0.213)$ \\
$\mathbf{4 5}$ & $24.6(23.2,26.0)$ & $24.7(23.3,26.1)$ & $22.0(20.6,23.3)$ & $22.1(20.7,23.4)$ & $-2.62(-4.51,-0.72)$ & $0.007(0.011)$ \\
$\mathbf{9 0}$ & $21.1(20.0,22.3)$ & $21.2(20.1,22.4)$ & $24.2(22.9,25.5)$ & $24.3(23.0,25.6)$ & $3.03(1.40,4.65)$ & $<0.001(0.001)$ \\
$\mathbf{1 3 5}$ & $22.7(21.4,24.0)$ & $22.8(21.5,24.1)$ & $22.0(20.5,23.5)$ & $22.1(20.6,23.6)$ & $-0.69(-2.61,1.24)$ & $0.482(0.557)$ \\
$\mathbf{1 8 0}$ & $26.2(23.8,28.7)$ & $26.3(23.9,28.8)$ & $18.7(15.9,21.5)$ & $18.8(16.0,21.6)$ & $-7.53(-11.17,-3.88)$ & $<0.001(<0.001)$ \\
\hline
\end{tabular}

Baseline and hindlimb suspended (HS)-induced IOP changes by age group (young, old), side (left, right) and time (0-180). All estimates are based on a linear-mixed effects model (assuming the random-effect $=0$ ). For example, the average IOP within the young male cohort at baseline (day 0) was $18.0 \mathrm{mmHg}(95 \% \mathrm{Cl}: 16.9-19.0)$ [left eye]. The similar value within old male cohort was 19.1 (17.7 - 20.6). The difference (old-young) in these average values was 1.19 (-0.51-2.89). Given side was modeled as a main-effect only, the cross-sectional age group differences are the same for the left and right eye (e.g., 19.1-18.0=1.1 [day 0; left], 19.3-18.1=1.2 [day 0; right]; slight differences due to rounding).

Table 4. HS-induced IOP temporal changes by age cohort.

\begin{tabular}{ccccccc}
\hline $\begin{array}{c}\text { Temporal } \\
\begin{array}{c}\text { Comparison } \\
\text { (days) }\end{array}\end{array}$ & \multicolumn{2}{c}{ YM Cohort } & \multicolumn{2}{c}{ OM Cohort } & \multicolumn{2}{c}{$\begin{array}{c}\text { Change Comparisons by Age Group } \\
\text { (Old-Young) }\end{array}$} \\
\cline { 2 - 7 } & Estimate $\mathbf{( 9 5 \%}$ Cl) & p-value (p-FDR) & Estimate (95\% Cl) & p-value (p-FDR) & Difference (95\% CI) & p-value (p-FDR) \\
\hline $\mathbf{4 5}$ vs. $\mathbf{0}$ & $6.62(5.05,8.20)$ & $<0.001(<0.001)$ & $2.82(1.17,4.46)$ & $0.001(0.002)$ & $-3.81(-6.08,-1.53)$ & $0.001(0.002)$ \\
$\mathbf{9 0}$ vs. $\mathbf{0}$ & $3.19(1.96,4.41)$ & $<0.001(<0.001)$ & $5.02(3.43,6.61)$ & $<0.001(<0.001)$ & $1.83(-0.17,3.84)$ & $0.073(0.098)$ \\
$\mathbf{1 3 5}$ vs. $\mathbf{9 0}$ & $1.54(0.43,2.65)$ & $0.007(0.011)$ & $-2.17(-3.44,-0.91)$ & $0.001(0.002)$ & $-3.71(-5.40,-2.03)$ & $<0.001(<0.001)$ \\
$\mathbf{1 8 0}$ vs. $\mathbf{9 0}$ & $5.08(2.47,7.69)$ & $<0.001(<0.001)$ & $-5.48(-8.46,-2.49)$ & $<0.001(0.001)$ & $-10.55(-14.52,-6.59)$ & $<0.001(<0.001)$ \\
\hline
\end{tabular}

Hindlimb suspended (HS)-induced IOP changes compared to baseline (time -0 days) or during the normal posture recovery period compared to the end of HS (time - 90 days) measurement as well as rates of change comparison by age group (old-young). All estimates are based on a linear-mixed effects model (assuming the random-effect $=0$ ). For example, the average IOP change within the young male cohort between day 45 and day 0 was $6.62 \mathrm{mmHg}(95 \% \mathrm{Cl}: 5.05,8.20)$ after adjusting for side (left/right). The analogous value within the old male cohort was $2.82(1.17-4.46)$. The difference (old-young) in these rates of change was $-3.81(-6.08--1.53)$. 


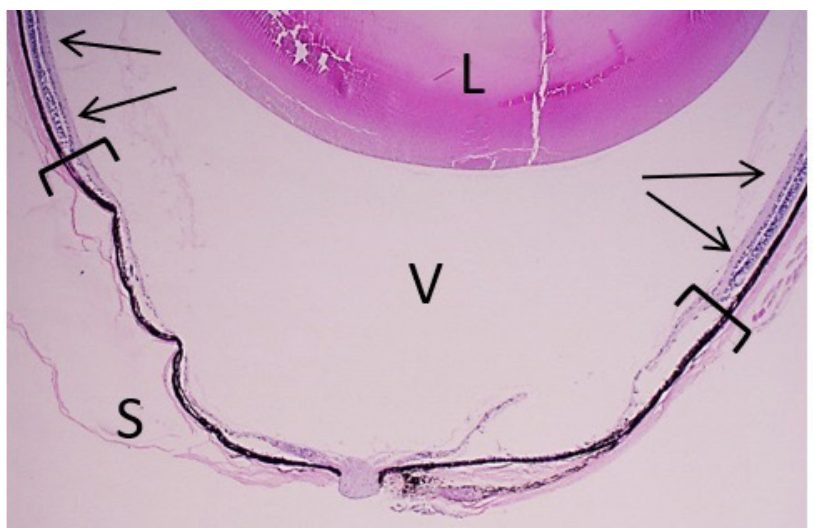

A
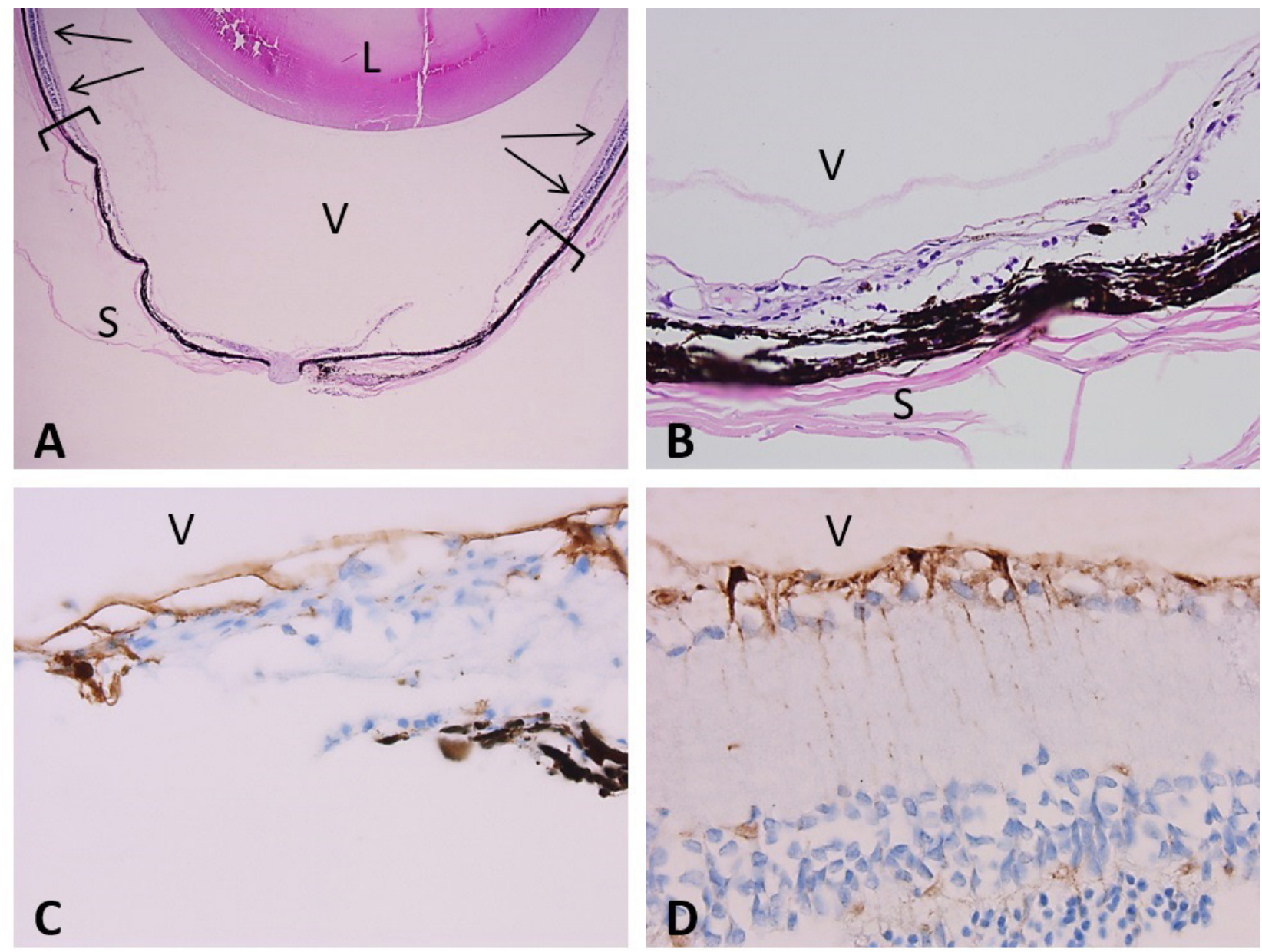

Figure 1. Histological examination of young female rat $\mathrm{HS}+90$ days recovery eye sections. (A) Low power magnification (2X) H\&E stain shows residual peripheral normal retina (arrows) with a posterior thin retina forming the chorioretinal scar. The lens (L), vitreous (V) and sclera (S) are unremarkable. (B) High magnification (20X) H\&E stain of a chorioretinal scar. (C) 40X magnification view of the retina in the scar immunostained with anti-GFAP antibody shows the irregular proliferation of glial cells in the inner layers (facing vitreous (V)). Immunohistochemistry, DAB chromogen, Glial fibrillary acidic protein antibody. (D) 40X magnification of the edge of the retinal scar immunostained with vimentin highlights the proliferating and activated Müller cells. Vitreous $(\mathrm{V})$. Immunohistochemistry, DAB chromogen, vimentin antibody.

implies that effect of HS was more rapid among YM versus YF. By day 90 , we were unable to detect differences in the rates of change between the sex groups $(0.35,-1.20-1.90)$.

From Table III, the average OS IOP at baseline was 18.0 (16.9-19.0) and 19.1 (17.7-20.6) in the YM and OM cohorts, respectively. Note, the estimates for the YM cohorts differ slightly since two separate models were constructed using two different comparator cohorts (YF or OM). This corresponds to an average baseline difference (OM-YM) of $1.19(-0.51$, 2.89). By day 45 , the average HS-induced OS IOP estimates increased to $24.6(23.2,26.0)$ and 22.0 (20.6-23.3) which corresponded to an average OS IOP difference of -2.62 $(-4.51,-0.72)[p=0.007, p-f d r=0.011]$. Among the YM cohort, the temporal change between day 45 and baseline was 6.62 $(5.05-8.20 ; p<0.001)$ [Table IV]. Similarly, among the OM cohort, this increase was $2.82(1.17-4.46)$. The differences in these rates of change was $-3.81(-6.08,-1.53)[p=0.001$, $p$-fdr $=0.002]$ which implies that effect of HS was more rapid among YM versus OM. By day 90, we were unable to detect differences in the rates of change between the age groups $(1.83,-0.17-3.84)$.

\section{Histopathology analysis of the retina reveals histologic alterations}

Histology and ocular pathology examination was performed in the retina sections stained with H\&E. An incidental finding 

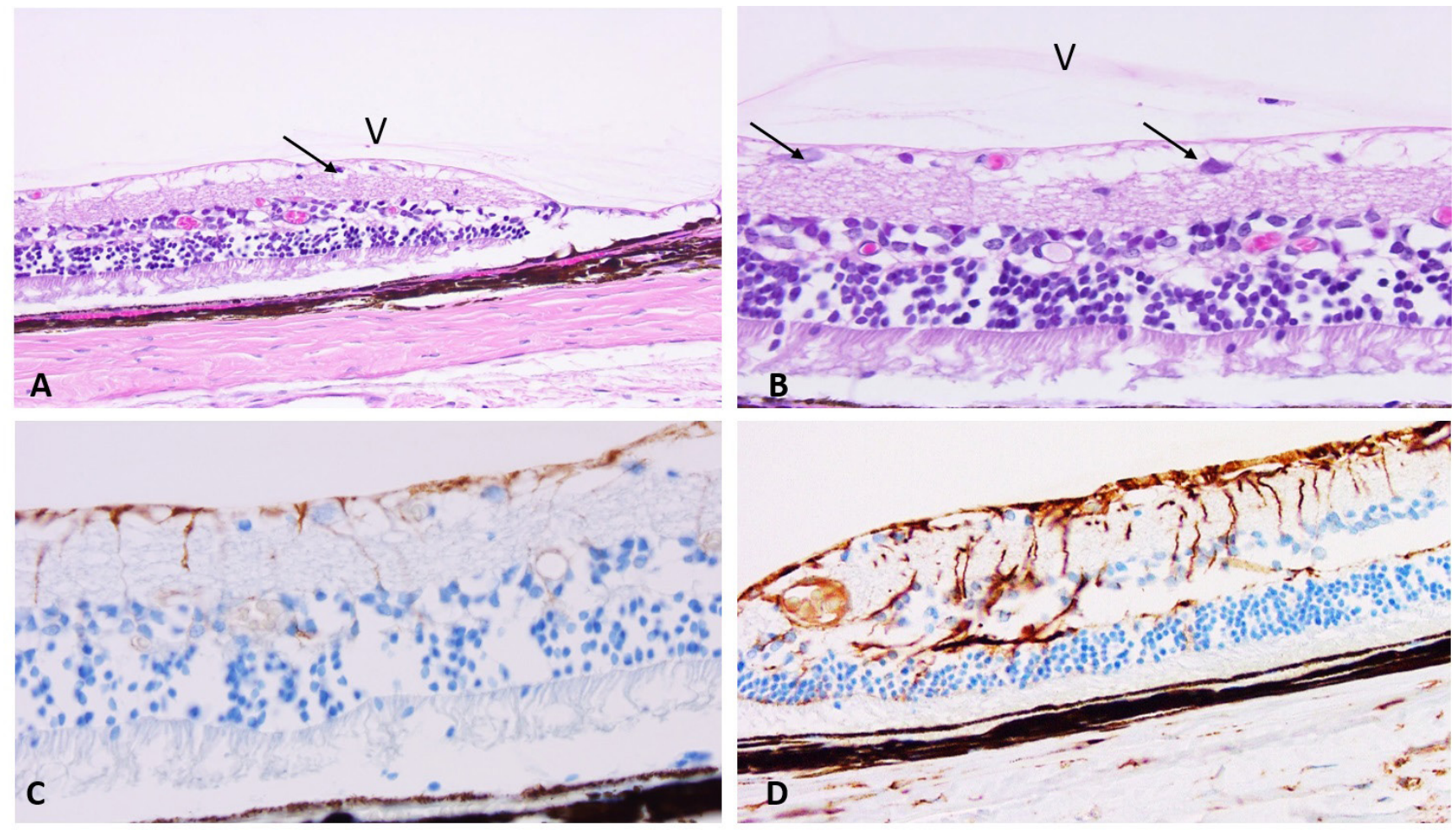

Figure 2. Histologic effects of HS in the rat retina of young male and female rats. (A) H\&E staining at 20X magnification of peripheral retina showing few inner layer ganglion cells (arrow) and some condensation of vitreous (V). (B) Images of retina from YM at 180 days at equator is thicker but also shows some drop in ganglion cells (arrow) and condensed band of vitreous (V). H\&E, original magnification 20X. (C) Immunohistochemistry example of GFAP staining of the glial and Muller cells in equatorial retina a male hind limb suspended (HS + 14 days recovery). Immunohistochemistry, DAB chromogen, GFAP antibody, 40X magnification. (D) Immunohistochemistry example of vimentin staining of Muller cells in the peripheral retina of a male hind limb suspended (HS+90 days recovery). Immunohistochemistry, DAB chromogen, vimentin antibody, 40X magnification.

was the presence of peripapilar chorioretinal scarring consistently localized around the $20^{\circ}$ region posterior to the optic disc. The abnormalities consisted of various degrees of severity and included photoreceptor loss, choroidal scarring, and retinal pigment epithelium (RPE) cell migration (FIG 1). In the most severe case, all neural layers of the retina were lost. The scars were also composed of varying degrees of gliosis with predominance of activated and proliferating Müller cells, some into the superficial choroid, and proliferation of the RPE. These features are highlighted by the GFAP staining of the glial and Müller cells (FIG 1 A-D). To some extent, from very mild to severe, all specimens seemed to be affected, regardless of experimental conditions. It is speculated that genetic shifting in the inbred colony may account for this phenotype. In view of this, the histology analysis was limited to the retina hemisphere free from these abnormalities.

The findings in the retina were more prominent in the equator and posterior retina. Peripheral retina is defined by the retina reaching the pars plana of the ciliary body and anterior to the equator. The equatorial retina is at the equator of the eye and the posterior and peripapillary retina is in the back of the eye around the optic nerve (FIG 1, A-B).

Retinal glial reaction was investigated by immunohistochemistry using GFAP which highlights activated astrocytes (see inner retina in perivascular location) and activated Müller cells. In addition, vimentin immunohistochemistry performed to highlight activated Müller cells, mostly manifested in the chorioretinal scars (FIG 1 C-D; 2 E-F). Outside of the chorioretinal scars, there was GFAP (between $1+$ and $2+$ ) staining in male $\mathrm{HS}$ rats decreasing from 14 days $\mathrm{HS}$ to $\mathrm{HS}+90$ days recovery after restoration to normal posture. In contrast, the female HS rats showed more reactive astrocytes in the inner retina in the $\mathrm{HS}+90$ days recovery $(2+)$ than in the 14 days $\mathrm{HS}(0.5+)$ (FIG 2 C-D). Transcriptionally, upregulation of GFAP mRNA was observed in young females at $\mathrm{HS}+14$ days recovery (2.9 fold, $p=0.01$ ) but not in the young males.

Müller cells appeared more prominent when stained with vimentin in suspended male rats and the change was dependent on length of time $(1+$ at 14 days $\mathrm{HS}$ versus $2+$ at 


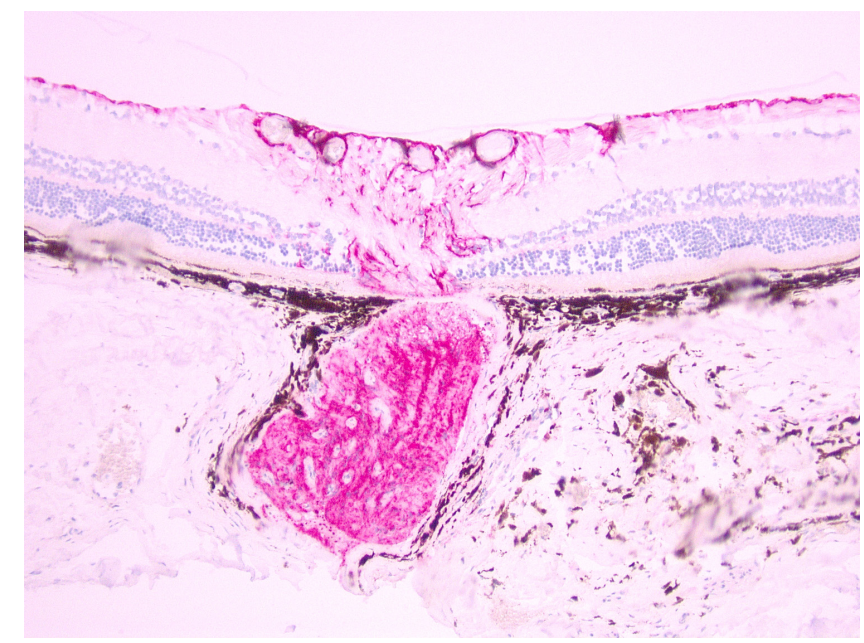

Figure 3. Histologic effects of HS in the rat optic nerve. (A) Image of a young male rat at 14 days $\mathrm{HS}$ at $40 \mathrm{X}$ magnification showing the posterior retina surrounding the optic nerve with intense immunoreactivity to GFAP antibody in the head of the nerve. Immunohistochemistry, Permanent Red chromogen, GFAP antibody.

90 days HS and HS +14 days recovery, versus $3+$ for the HS +90 days recovery). No normal posture male control rats had positive staining of Müller cells. The female HS rats had a mild response in the early periods ( $1+$ at 14 days HS versus $1.5+$ at HS +14 days recovery). However, there was a 1-2+ staining of the Müller cells in some of the control female rats $(2+$ in the older group of 180 days). This probably is related to a more prominent chorioretinal scarring in this same group and may be an extension (edge effect) of the lesion. (FIG 2 E-F).

Not all the sections of the eyes contained optic nerve, but from those present, the YM suspended rats showed heavy GFAP $4+$ staining at 14 days HS of the astrocytes within the optic nerve at the head and scleral portions, $3+$ for HS +14 days recovery and $1+$ for the HS + 90 days recovery. The female suspended rats showed $2+$ in the HS +90 days recovery only. The control male rats showed a $1+$ staining at the level of the disc/head of the nerve at HS +14 days recovery and $\mathrm{HS}+90$ days recovery, while the female controls showed $1+$ in the same area at 14 days HS only (FIG 3).

\section{Transcriptomic analysis reveals sex and age dependent key regulators}

Microarray gene expression analysis of retinas from the various experimental groups is showed in FIG 4 as a principal component analysis (PCA) plot. Three principal components account for nearly $45 \%$ of the variance in all experimental groups, however no clear separation of the treatment effect was seen when all groups are considered. In contrast, a clear separation was noticed between retinas from females and males, indicating that the transcriptional landscape of the retina is sex dependent, regardless of treatment, especially along Principal Component 1 (PC1). In addition, when all groups are identified individually, the PCA plot shows clustering of the YM, YF and OM cohorts, suggesting that age has an effect along PC2 and PC3. Interestingly, when performing paired comparisons between control and HS retinas for each experimental cohort and time points, a clear effect of the HS treatment was evidenced with a preponderant effect of one principal component accounting for approximately $70 \%$ of the variance.

Pathway analysis was subsequently performed to investigate the molecular mechanisms, cellular processes and gene networks involved in the responses to HS, which were then compared among the groups (Supplementary FIG 1). Both common and unique pathways, upstream regulators and key driving genes were identified in the various experimental groups across gender, age and length of treatment or recovery periods. As an example, differential gene expression across the sexes in response to HS was reflected in mechanisms and key regulator genes that differed between females and males. Likewise, age seemed to incorporate an important factor in the response, judging by a set of key driving genes in old males in response to HS. Interestingly, all analyses supported that YM at advanced age (104 days collection point) resembled OM in their expression response profile.

A clear variation in the transcriptional response in the female and male cohorts was observed across the length of the HS treatment. Hierarchical cluster analysis of microarray genes and upstream regulators (FIG 5) showed that YM at HS +14 days recovery (104 day) behaved more similarly to OM at 14 days HS and HS +90 days recovery (180 day), suggesting that age probably is a predominant factor in modulating the response to HS at longer time points. This clustering was also observed for canonical pathways (Supplementary FIG 2). The age effect was specifically observed at 14 days, for which the pattern of miRNA inferred pathways showed an opposite trend between YM and OM (Supplementary FIG 3).

In order to identify the common response to HS in the various groups without the confounding response to the recovery period, we first limited our pathway analysis to the HS treatment at 14 and 90 days. The main upstream regulators identified according to prevalence of change are shown in FIG 6. The proto-oncogene TP53 was identified as a common upstream regulator for processes in all three cohorts both in the short (14 day) and long-term response (90 day). Other upstream activators that were relevant were the mineralocorticoid receptor $\mathrm{NR} 3 \mathrm{C} 2$, the zinc finger transcription factor SNAIL1, and hepatocyte growth factor HGF. Key genes were then investigated in these networks using similar methods as described in previous work (Beheshti et al., 2015, Beheshti et al., 2015, Beheshti et al., 2018). Of the key genes identified as relevant in the 
A

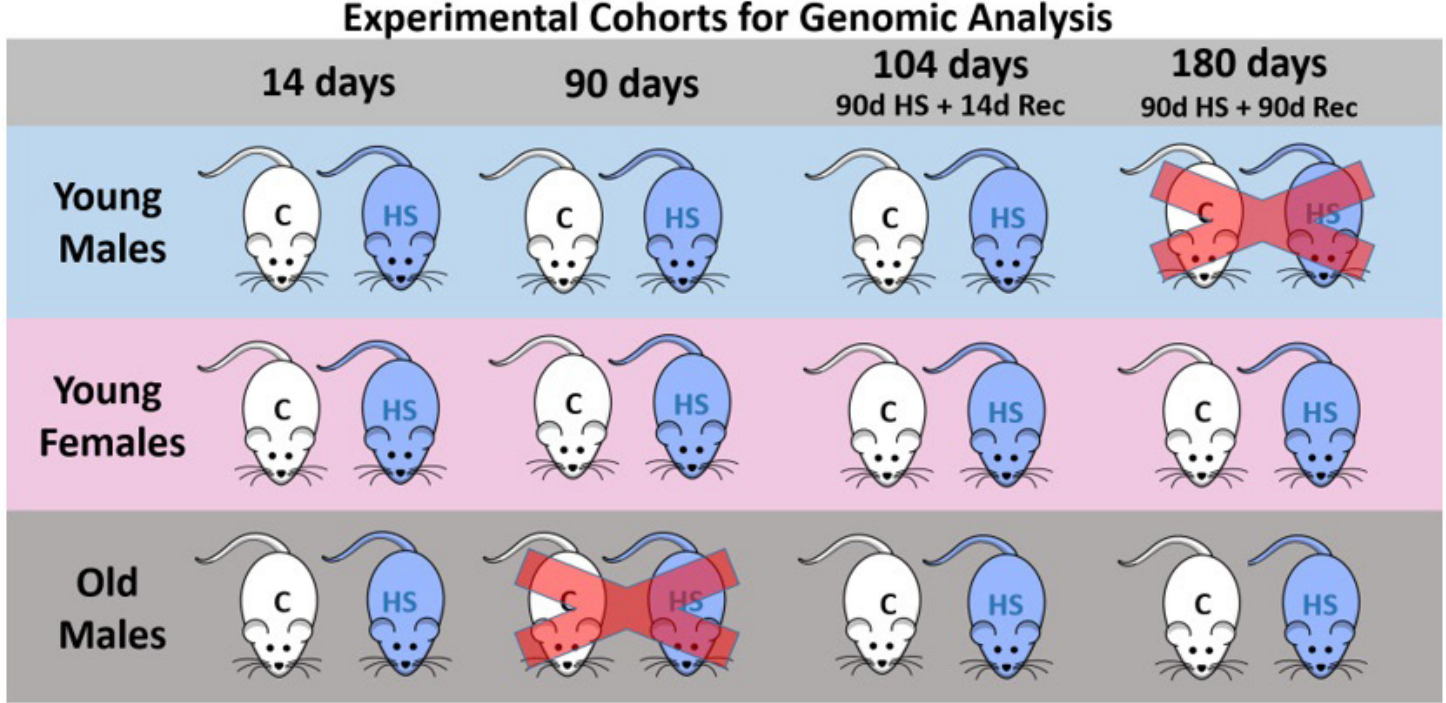

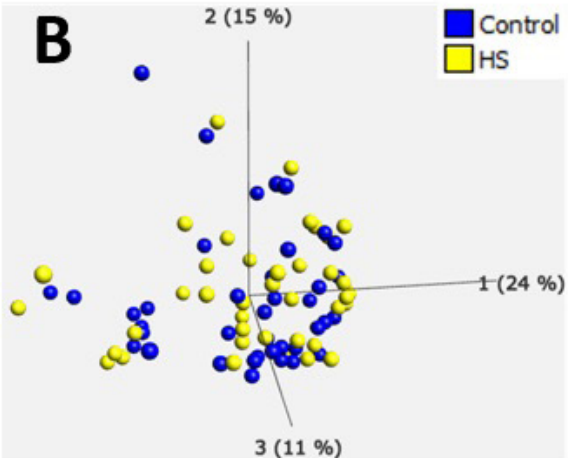

Treatment - HS vs C

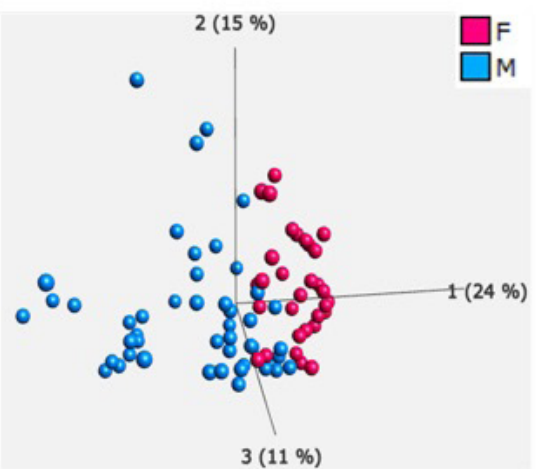

Gender - Females vs Males

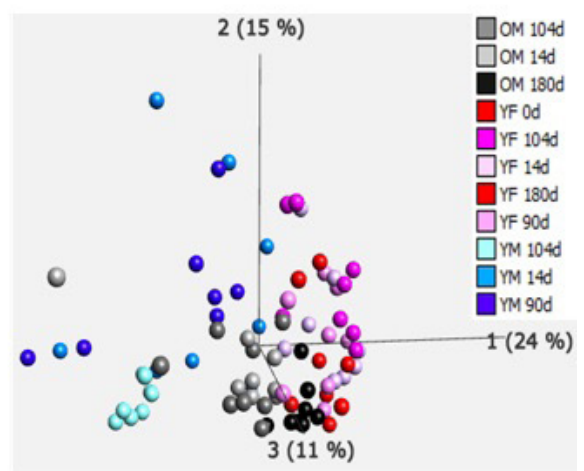

Cohort - YM, YF, OM

c

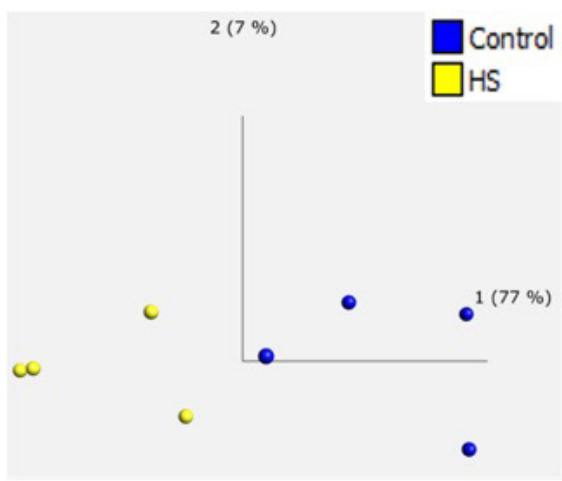

YM at 14 days HS

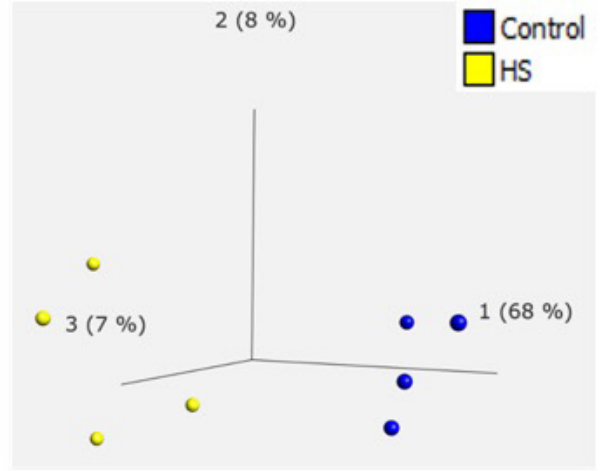

YF at 14 days HS

Figure 4. Microarray analysis of rat experimental cohorts. (A) Experimental groups represented in the microarray analysis. Groups for which no retinas were available are marked with a red X. (B) Principal component analysis (PCA) of gene expression in all experimental groups shown in A. Left, color coded for treatment; middle, color coded for gender; right, color coded by cohort (YM, YF, OM). (C) PCA showing clustering by treatment of YM and YF at 14 days of treatment. 
FDR $<0.05$

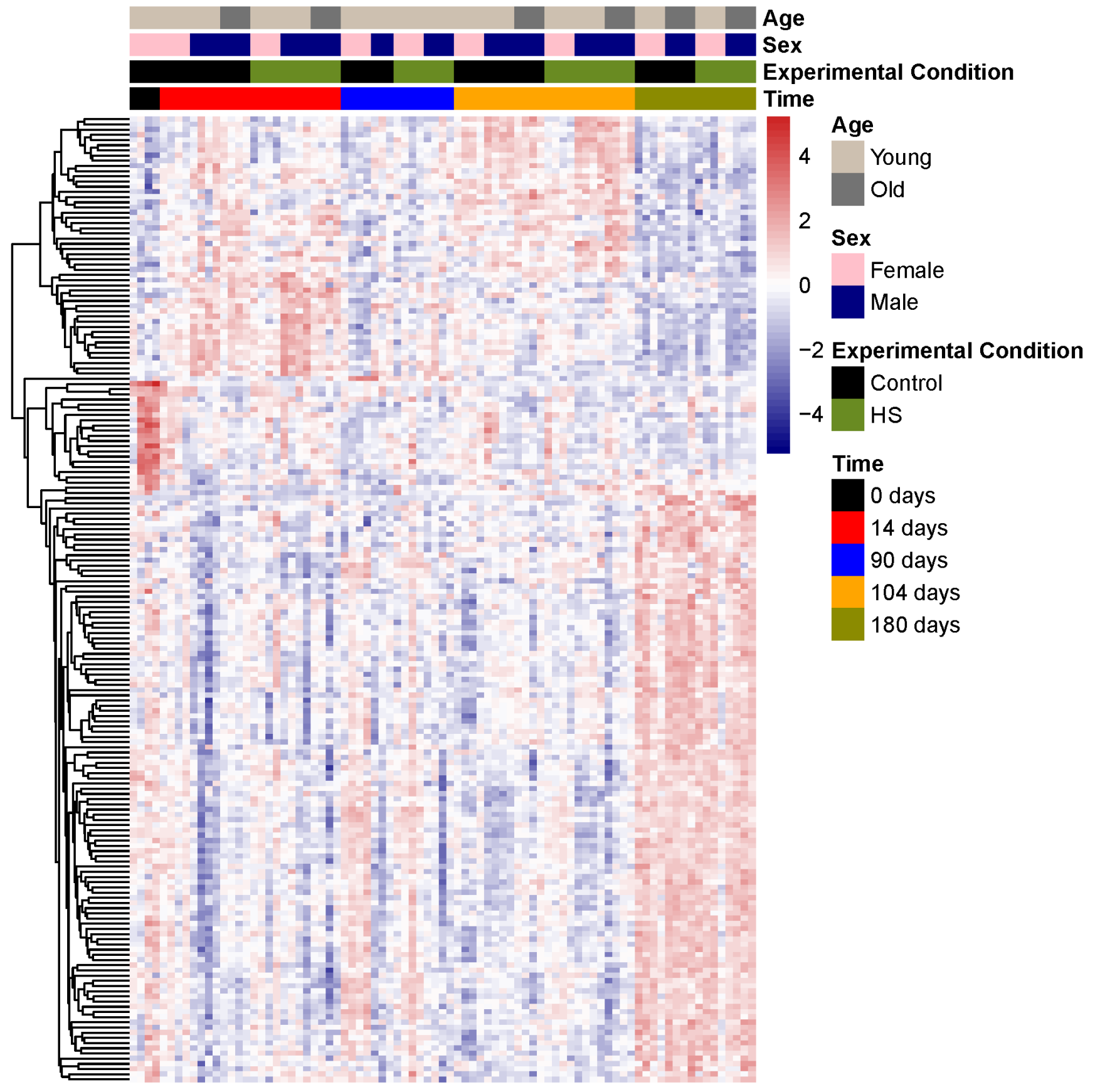

Figure 5. Heatmap of the significantly regulated genes (FDR < 0.05). Euclidean clustering was used for clustering the genes.

response to HS, CSKN1A1 was found in all three cohorts, suggesting it may hold a leading role as a key regulator in the response. Interestingly, CSKN1A1 acts upstream TP53 in pathways related to cell death and survival (FIG 7). Among the differentially expressed genes with the largest expression change, the transcription factor, Egr1, was upregulated (3.96fold, $p=0.002$ ) in the early response time (14 day) in young males but not females, constituting an example of the various sex-dependent HS-differentially expressed genes. Another gene significantly upregulated 3.95 -fold, $(p=0.007)$ in $Y M$ at 


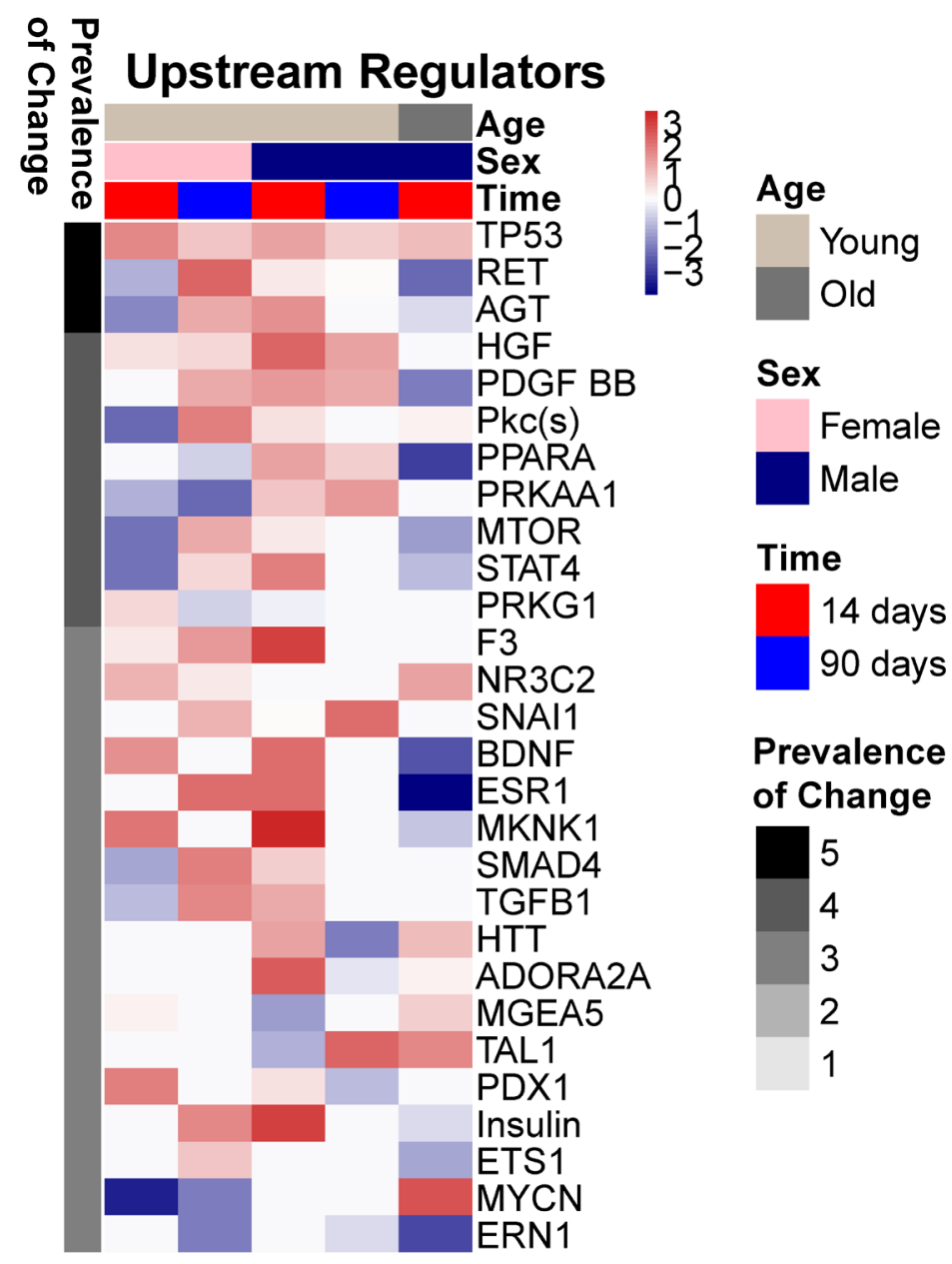

Figure 6. Upstream regulator analysis utilizing Ingenuity Pathway Analysis (IPA) on the significantly regulated genes (FDR < 0.05$)$. The results are shown only for the common upstream regulators across 14 and 90 days as a heatmap of the activated z-scores.

14 days was deiodinase 2 (DiO2), an enzyme that converts $\mathrm{T} 4$ (thyroxine) to active T3 (triiodothyronine). The complete gene expression data is available from the NASA Life Sciences Database (https://lsda.jsc.nasa.gov) and Genelab (www. nasa.gov/genelab) databases.

Regardless of treatment, gene expression in the retina of males and females was different across all groups. Variance increases in ANOVA using Qlucore identified the gene coding for elF2s3y (a eukaryotic translational initiation factor implicated in eye development) to be expressed differently in females and males with the highest statistical significance $(p=5.57 \times 1005, q=0.0001)$, as expected for a $Y$ chromosomelinked gene, supporting our gender comparison analysis. . In agreement with this finding, the key genes involved in the differential response pointed to an enhanced representation of translation associated genes in females at 14 days of treatment compared to males (FIG 6).
The differential gene expression response appeared particularly "active" judging by the number of differentially expressed genes (DEG) and key driving genes at collection time points closest to the posture transitions (14 days and 104 days), while less DEG were identified at the end of the treatment period (90 day) (FIG 7 and Supplementary FIG 4). This suggested that some degree of adaptation to the simulated microgravity conditions had taken place. Note that samples were not available from the YM 180 day and OM 90 day groups, therefore limiting the possibility of a complete comparison among all the groups. In particular, YM and OM at 14 days show evidence of immune and inflammatory related pathways. Specifically, an activated CXCR4 pathway was apparent in young males and a TGF $\beta$ pathway involvement at 104 days in both males and females. The Wnt- $\beta$-catenin pathway was also represented based on the finding of key genes, namely FZ4 (YF 104 day) and CTNNB1 (OM 14 day). 


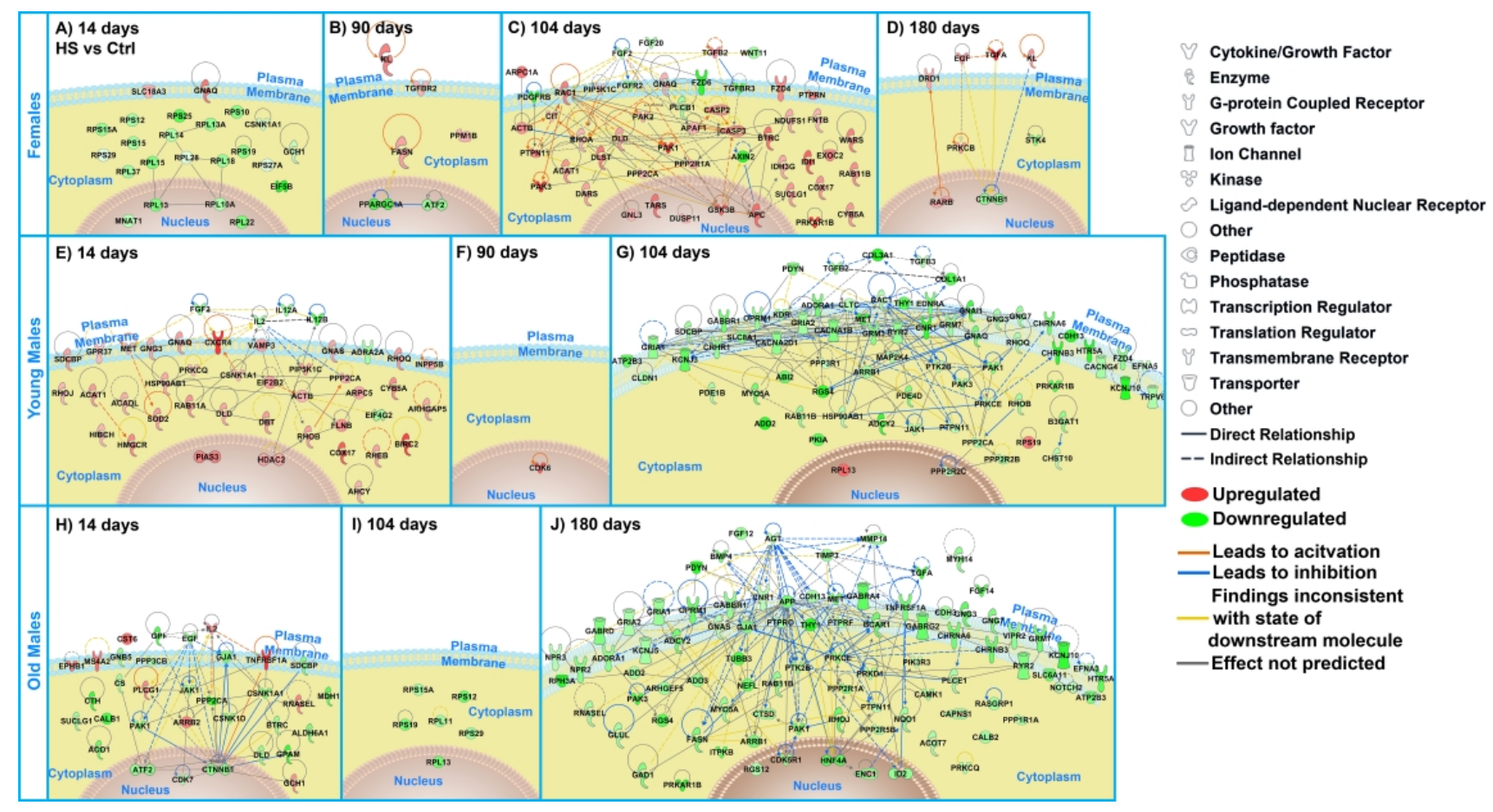

Figure 7. Network diagram of "Key Genes" comparing HS vs controls for females for (A) 14 days, (B) 90 days, (C) 104 days, and (D) 180 days. For young males for (E) 14 days, (F) 90 days, and (G) 104 days. For old males for (H) 14 days, (I) 104 days, and (J) 180 days. Transcriptionally upregulated genes in red, downregulated genes in green and connectivity with predicted relationships between these key genes are displayed using Ingenuity Pathway Analysis software. The figure legend indicates the line relationship with solid lines representing known direct relationships between the genes and dash lines representing the known indirect relationships. The arrows in the networks represent which direction the genes are acting on each other.

Of particular note, the observed gene networks, canonical pathways and key gene regulators directly pointed towards neurodegenerative processes occurring at later time points (104 and 180 days). Specifically, OM at 180 days showed evidence of marked neurodegeneration affecting ion transport and neuronal survival (e.g., b-amyloid precursor protein (APP) appears centrally localized in the mentioned networks) (FIG 6 and Supplementary FIG 1).

\section{DISCUSSION}

We hereby report the results of the first study evaluating the effects of HS in the rat retina in an effort to investigate whether significant changes occur in response to a treatment that has been considered an analog of microgravity for many years. While HS represents a valid model for animal analog microgravity studies with respect to certain physiologic systems, in particular muscle and bone, the main aim for this study was to determine whether retinal alterations would ensue as a result of the fluid redistribution resulting from the positional change. SANS signs found in crewmembers do not permit an assessment of the cellular effects of the stress associated with the condition. However, the accessibility to tissue from the rats in this study allowed to focus on the molecular and histologic effects in the retina in a simulated microgravity environment.

\section{Transcriptional sexual dimorphism}

There is limited evidence suggesting that sex hormones can influence the development of certain retinal disorders and/ or the protection against them (Nuzzi et al., 2018, Getter et al., 2019). Sex steroid receptors are present throughout the eye and, in addition, estradiol and testosterone are locally produced in ocular tissues. Sex hormones can have a neuroprotective action on the retina and modulate ocular blood flow (Jiang et al., 2019). Recent research into sex-related differences has begun to reveal possible links between estrogens and eye diseases, i.e., glaucoma, agerelated macular degeneration (AMD), diabetic retinopathy and cataracts (Grover 2017). There is a slightly higher prevalence of vision impairment in females over males, as reported by 
US epidemiologic records (Grover 2017). These differences have been ascribed to reporting and health care bias rather than to actual differences in the propensity to eye diseases between sexes. The transcriptional landscape in the retina of female and male rats in this study was substantially different. The fact that a notable sex-dependent gene expression profile was observed in our experiment may support the concept that there may be different responses to stress in the retina of females compared to males. However, sexual dimorphism in the retina (as reported in this study), brain and other tissues cannot easily be explained merely by gonadal steroid action. Endogenous synthesis of testosterone and estradiol occurs in the retina, as well as expression of estrogen receptors $\alpha(E r \alpha)$ and $\beta(\operatorname{Er} \beta)$ (Cascio et al., 2015) therefore, the possibility that the local steroid hormone environment takes precedence over the systemic hormonal environment should not be ignored.

When increasing the bioinformatics variance to render the gender-specific genes selection more stringent, it was possible to inquire about which genes seem to be more dependent on gender. We identified specific types of crystallin, crystallin $y d$ and $e$ (data available from the entire gene expression record in the Genelab repository), and a translation initiation factor, elF2s subunit $3 y$. Crystallins are major proteins in the lens, responsible for its transparency and refractive properties. However, they are also expressed in the retina and other tissues (Templeton et al., 2013), where they exert a cytoprotective function against protein misfolding. Gender differences have previously been reported in crystallin expression in the lens (D'Anna et al., 2011), in agreement with our own results in the retina (Guest et al., 2006).

We also found that elF-2y is another gene with dimorphic expression in the retina. The translation initiation factor elF2s3y is reportedly involved in eye function and development (Ehrmann et al., 1998). The gene coding for elF2s3y is located on the $Y$ chromosome and its homologue in the $X$ linked chromosome. It is reported to evade $X$ inactivation and thus its expression level is different in males and females and not fully characterized (Xu et al., 2002). Much like its previously reported expression in the brain (Xu et al., 2002), its expression in the retina might explain differences in the retinal biology of males and females, in addition to the abovementioned contribution of the sexual steroids.

Rodent studies have shown that testes size, spermatogenesis and testosterone levels are reduced following 7 days of HS (Deaver et al., 1992, Hadley et al., 1992), along with an increase in luteineizing and FSH hormones. In spaceflight, spermatogenesis in rats is normal, but testosterone levels decrease after 2 weeks (Amann et al., 1992). It is therefore important to consider how the endocrine milieu resulting from HS affects the retina, since it is known that both estrogen and androgen receptors are expressed and sex steroid synthesis occur locally in the retina (Cascio et al., 2015). Of note, neither estrogen receptor $\alpha$ nor $\beta$ showed as significantly regulated genes throughout all groups and conditions in this study (data not shown). Weighing the relative contribution from the systemic and local sex steroid environment in the retina is a topic that deserves further investigation

In summary, we can recognize three factors that may contribute to the differential response to HS in the retina: the different dosage of elF2 $\gamma$ and its expression in the retina, the local sexual steroid milieu, and the alteration of steroid homeostasis due to the suppression of testosterone elicited by HS.

\section{Transcriptional response to hindlimb suspension in the rat retina}

The results of this work reveal both commonalities and dissimilarities in the response to HS in the rat retina of the different cohorts based on age, sex, and length of treatment. This is supported by evidence of different key regulators and cellular processes observed through gene enrichment analysis (FIG 5, FIG 6, and Supplementary FIG 1).

If we consider the YM as the reference cohort, specific responses were observed that are worthy of mention. A marked upregulation of nearly 4-fold of the transcription factor Egr1 occurred at 14 days of treatment, which was not observed in YF or OM cohorts. Egr-1 is a stretch-activated transcription factor which is significantly increased in patients with pulmonary arterial hypertension (PAH) (Kyriakou et al., 2019). PAH is a pulmonary angioproliferative disease with high morbidity and mortality, characterized by a typical pattern of pulmonary vascular remodeling including neointimal lesions (van Albada et al., 2010). Neointima scar tissue forms within blood vessels, resulting in reduced lumen in the arterial vessels and can develop with damage to the arterial wall, followed by platelet aggregation at site of injury, recruitment of inflammatory cells, proliferation and migration of vascular smooth muscle cells, and collagen deposition. Since most of these pathways appear represented in the canonical pathways inferred from the YM data, it is plausible to hypothesize the following process in YM as a result of HS: The fluid shift accompanied by vessel wall stretching would lead to Egr1 upregulation, which in turn could trigger inflammation and wall thickening by a process similar to PAH. CREB pathway activation was also congruent with Egr1 upregulation in YM. In summary, signaling components implicated in Egr-1 expression in YM are suggestive of potential vascular pathophysiology (Cheyou et al., 2014). At this point, an explanation for the Egr1 differential expression between males and females is elusive. It is reported that sexual dimorphism exists in PAH, which would be in agreement with the observed sexual dimorphism in retina gene expression in our study. However, as already explained above, the characterization of the sex 
hormonal retinal medium is not fully understood in view of the confounding factors that may contribute to the differential response to HS in the retina. Of note, no Egr-1 upregulation was observed in a previous study in C57BL/6J mice flown for 13 days in low-Earth orbit (STS-135 shuttle mission) (Theriot 2015), which, under the hypothesis presented, could be explained by a more pronounced cephalad fluid shift in head down tilt of HS compared to microgravity. Additionally, Egr-1 upregulation cannot be ignored from consideration from stress exerted in the axons of the retinal ganglion cells (Yasuda et al., 2014).

The Wnt signaling pathway plays a critical role in retinal development, with regards to both angiogenesis and neurogenesis. The pathway analysis in this study indicated that the Wnt signaling pathway activity is decreased in HS in both females and males (SUPPLEMENTARY FIG 2). This is quite a surprising finding according to the histologic phenotype observed in HS retinal sections which showed condensed vitreous bands in the inner part of the retina and the presence of unusual epiretinal cells (FIG 1 and 2), resembling the pathology of proliferative vitreoretinopathy (PVR). PVR is a condition that develops as a complication of retinal detachment (Sadaka and Giuliari 2012). It is characterized by vitreous retraction and periretinal proliferation of RPE cells and glial cells. The RPE cells undergo epithelial mesenchymal transition (EMT) and migrate into the vitreous. The adhesion between RPE, neural retina and extracellular matrix (ECM) is lost. Migratory RPE cells secrete fibrotic membranes (ECM laid on the vitreous side is referred as epiretinal membranes), that contracts and pulls the retina that can lead to folds and (secondary) detachment. Intraocular inflammation is a predisposing factor for PVR and as such, TGF $\beta 2$, as well as interleukins like IL6, TNF $\alpha$ and PDGF may all be involved in the pathology of the condition. A pro-inflammatory environment seems to be the case for the retina of rats in HS. The Wnt-gene network is involved in EMT, via activation of HGF (Jiang et al., 2007, Pan et al., 2010, Huang et al., 2019). Interestingly, our results show that HGF is activated according to the upstream regulators analysis (FIG 6). RPE cells express HGF receptor and HGF stimulates RPE cell migration, possibly explaining the presence of RPE cells in the epiretinal space. This is quite an interesting finding that elicits some speculation. The exemplifying images in FIGURE 1 and FIGURE 2, show some retinal sections with condensed vitreous bands in the inner part of the retina and the presence of unusual epiretinal cells resembling the pathology of proliferative vitreoretinopathy.

The 14-day timepoint is particularly relevant since it may highlight the early responses to HS, whereas the 90day timepoint may reflect that homeostatic mechanisms have already taken place and given way to adaptation to the environment of simulated microgravity. Indeed, the number of
DEG around the transition time points (14 and 104 days) was larger than the DEG at 90 days (SUPPLEMENTARY FIG 4).

Focusing on the common pathways and regulators across all cohorts at 14 days HS, CSNK1A1 was shown to be implicated in the three cohorts, downregulated in YF and $\mathrm{OM}$ and upregulated in YM. Interestingly, CSNKA1A acts upstream TP53, which is activated in all three groups. TP53 upregulation suggests that cell death and survival mechanisms may be involved in the response. Relationship analysis by STRING (FIG 8) allowed the identification of a direct relationship between CSKNA1A, TP53 and Egr1, which could explain the unique response of Egr1 seen in the YM cohort, consistent withCSKNA1A and TP53 upregulation.

As mentioned in the Results section, the response to HS was dependent on age. The analysis of the age effect in the retina is paramount in distinguishing the effects of senescence from specific pathologic processes in age-related ophthalmic diseases. Since the experimentation in this study spans 180 days, it would be expected that gene expression and histologic changes would occur in the retina of the rats within this period, especially, between the YM and OM groups. The age effect based on gene expression analysis in the retinas of YM and OM may correlate with a comprehensive list of anatomic and functional changes as previously reported for albino and pigmented rat strains (Nadal-Nicolas et al., 2018). These changes refer to thinning of the retina and a decrease in the cone population, associated with a decline in retinal function assessed by electroretinography. As shown in SUPPLEMENTARY FIGURE 1, numerous gene enrichment sets derived from our microarray data correspond to neuronal function, synaptic transmission and ion transport. In particular, the synaptic transmission set showing downregulation in the older animals (OM, and YM and YF at 180 days) may evidence a shift in the photoreceptor population as mentioned above. These observations warrant an in-depth analysis for their possible implication on SANS in further work and extend beyond the scope of this study.

The evidence of eye changes in rodents exposed to the spaceflight environment has been scarce until recently, thanks to the more frequent access to spaceflight animal experimentation on shuttle and the ISS. This paucity of studies refers mostly to rats, due to their larger size compared to mice. A recent study by Mao et al provides a thorough proteomic survey of the molecular changes in the retina of C57BL/6 male mice maintained in space for 35 days, showing evidence of blood brain barrier disruption, cellular loss and changes in crystallin expression, as well as a decrease in IOP at the time of return to Earth (Mao et al., 2019). A loss in retinal endothelial cells was also observed in mice after 7 days of HS (Mao et al., 2019). Overbey et al reported microCT imaging in C57BL/6 mice after a 35-day spaceflight surprisingly showed a thinning of the retina, RPE and choroid 


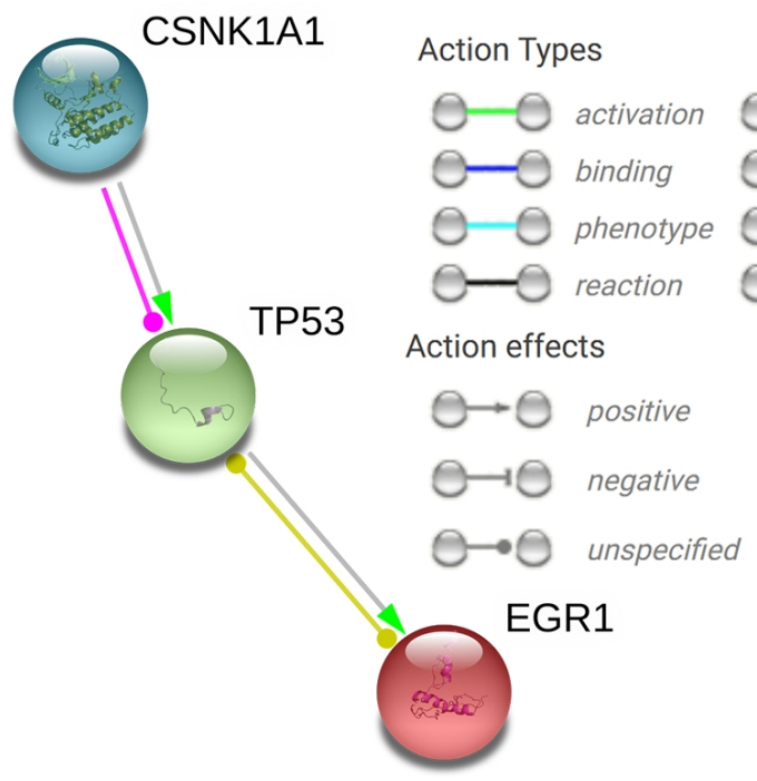

Figure 8. The network view of the key related genes CSNK1A1, TP53, and EGR1 interaction predicted using STRING. The figure legend displays the meaning of the line colors and symbols.

compared to ground controls, an observation that must bear in mind the stress of processing the samples approximately 38 hrs after splashdown (Overbey et al., 2019, Roque-Torres et al., 2020). Unfortunately, due to an incomplete record, our HS study was not able to provide data for comparison with the latter. However, as already mentioned and albeit the limitations faced by this ambitious experiment, our study is the first to document a comprehensive catalog of molecular and histologic changes in the retina of rats in HS compared to controls, across sex and age.

\section{CONCLUSIONS}

The results from this study provide transcriptional evidence in support of a response to HS in the rat retina that is affected by age and sex. Several pathways were identified that suggest that stress is imposed by the HS treatment, affecting the retinal vasculature, oxidative and inflammation status, RPE function, and glial activation, all of which may contribute to retinal senescence (Nadal-Nicolas et al., 2018). When interpreting the effects of stress on the retina, it is important to note that a different response may imply a different susceptibility or resilience to stressors (e.g. hypoxia, mechanical stress, inflammation, etc.) between males and females. The most significant genes showing gender-specific expression appear to be involved in cytoprotection, eye development and function, and may explain gender-dependent vulnerabilities to certain eye diseases.
It is important to note that, according to previous literature, HS induces suppression of testosterone production and spermatogenesis arrest in male rodents. Thus, the effects of HS in the two genders should be taken with caution since there may be confounding factors, namely, a) direct effects on the retina, and $b$ ) effects mediated by the change in steroid levels, which in turn may alter the steroid homeostasis in the retina.

Simulated microgravity by HS also induced gene expression changes in the retina that indicate stress conditions possibly contributing to cell loss, involving the CSNK1A1TP53 pathway. Other pathways indicative of effects in the neural retina included glial activation and, in the older males, increase of neurodegeneration markers like $\beta$-amyloid. The gene expression profile was also affected by age, as shown by the shift observed in males, where the YM entering the $104^{\text {th }}$ day of the experimental protocol resembled some of the characteristics of the baseline older male cohort.

Finally, with respect to IOP, different trends were observed in YM, YF and OM in response to HS. This could be investigated further as an added factor influencing the transcriptional responses in the retina.

It is important to note the limitations of this study with regards to number of animals per group, the attrition of samples for specific measures and groups and the incidental finding of retinal abnormalities in this rat strain. In spite of that, this study contributes interesting observations pertaining to the ocular response in a commonly used model of microgravity, in particular with reference to a currently intriguing condition 
observed in spaceflight, pioneering the investigation of the molecular and cellular impacts of microgravity in the eye.

\section{ACKNOWLEDGMENTS}

This material is based upon work supported by the National Aeronautics and Space Administration under grant NNX15AW48G. We would like to acknowledge Dr. Charles Fuller and his group at the University of California Davis for the execution of the animal experiment and tissue and data sharing.

\section{DISCLOSURE STATEMENT}

No competing financial interest exist.

\section{REFERENCES}

Almasieh M and Levin LA (2017). Neuroprotection in Glaucoma: Animal Models and Clinical Trials. Annu Rev Vis Sci 3: 91-120.

Amann RP, Deaver DR, Zirkin BR, Grills GS, Sapp WJ, Veeramachaneni DN, Clemens JW, Banerjee SD, Folmer J, Gruppi CM and et al. (1992). Effects of microgravity or simulated launch on testicular function in rats. J Appl Physiol (1985) 73:(2 Suppl): 174S-185S.

Beheshti A, Benzekry S, McDonald JT, Ma L, Peluso M, Hahnfeldt $P$ and Hlatky $L$ (2015). Host age is a systemic regulator of gene expression impacting cancer progression. Cancer Res 75:(6): 1134-1143.

Beheshti A, Neuberg D, McDonald JT, Vanderburg CR and Evens AM (2015). The Impact of Age and Sex in DLBCL: Systems Biology Analyses Identify Distinct Molecular Changes and Signaling Networks. Cancer Inform 14: 141-148.

Beheshti A, Ray S, Fogle H, Berrios D and Costes SV (2018). A microRNA signature and TGF-beta1 response were identified as the key master regulators for spaceflight response. PLoS One 13:(7): e0199621.

Bolstad BM, Irizarry RA, Astrand M and Speed TP (2003). Acomparison of normalization methods for high density oligonucleotide array data based on variance and bias. Bioinformatics 19:(2): 185-193.

Cascio C, Deidda I, Russo D and Guarneri P (2015). The estrogenic retina: The potential contribution to healthy aging and age-related neurodegenerative diseases of the retina. Steroids 103: 31-41.

Cheyou ER, Youreva V and Srivastava AK (2014). Involvement of the early growth response protein 1 in vascular pathophysiology: an overview. Indian J Biochem Biophys 51:(6): 457-466.

D'Anna C, Cascio C, Cigna D, Galizzi G, Deidda I, Bianchi L, Russo D, Passantino R, Bini L and Guarneri P (2011). A retinal proteomicsbased study identifies alphaA-crystallin as a sex steroid-regulated protein. Proteomics 11:(5): 986-990.
Deaver DR, Amann RP, Hammerstedt RH, Ball R, Veeramachaneni DN and Musacchia XJ (1992). Effects of caudal elevation on testicular function in rats. Separation of effects on spermatogenesis and steroidogenesis. J Androl 13:(3): 224-231.

Ehrmann IE, Ellis PS, Mazeyrat S, Duthie S, Brockdorff N, Mattei MG, Gavin MA, Affara NA, Brown GM, Simpson E, Mitchell MJ and Scott DM (1998). Characterization of genes encoding translation initiation factor elF-2gamma in mouse and human: sex chromosome localization, escape from X-inactivation and evolution. Hum Mol Genet 7:(11): 1725-1737.

Getter T, Suh S, Hoang T, Handa JT, Dong Z, Ma X, Chen Y, Blackshaw $S$ and Palczewski K (2019). The selective estrogen receptor modulator raloxifene mitigates the effect of all-trans-retinal toxicity in photoreceptor degeneration. J Biol Chem 294:(24): 9461-9475.

Globus RK and Morey-Holton E (2016). Hindlimb unloading: rodent analog for microgravity. J Appl Physiol (1985) 120:(10): 1196-1206.

Grover LL (2017). Making Eye Health a Population Imperative: A Vision for Tomorrow-A Report by the Committee on Public Health Approaches to Reduce Vision Impairment and Promote Eye Health. Optom Vis Sci 94:(4): 444-445.

Guest PC, Skynner HA, Salim K, Tattersall FD, Knowles MR and Atack JR (2006). Detection of gender differences in rat lens proteins using 2-D-DIGE. Proteomics 6:(2): 667-676.

Hadley JA, Hall JC, O'Brien A and Ball R (1992). Effects of a simulated microgravity model on cell structure and function in rat testis and epididymis. J Appl Physiol (1985) 72:(2): 748-759.

Huang AS, Stenger MB and Macias BR (2019). Gravitational Influence on Intraocular Pressure: Implications for Spaceflight and Disease. J Glaucoma 28:(8): 756-764.

Huang F, Chen J, Lan R, Wang Z, Chen R, Lin J and Fu L (2019). Hypoxia induced delta-Catenin to enhance mice hepatocellular carcinoma progression via Wnt signaling. Exp Cell Res 374:(1): 94-103.

Jiang D, Li T, Mao R, Du C and Liu J (2019). Deep Learning Based Dosimetry Evaluation at Organs-at-Risk in Esophageal Radiation Treatment Planning. Annu Int Conf IEEE Eng Med Biol Soc 2019: 868-871.

Jiang Y-G, Luo Y, He D-I, Li X, Zhang L-I, Peng T, Li M-C and Lin $\mathrm{Y}-\mathrm{H}$ (2007). Role of Wnt/ $\beta$-catenin signaling pathway in epithelialmesenchymal transition of human prostate cancer induced by hypoxia-inducible factor-1a. International Journal of Urology 14:(11): 1034-1039.

Kyriakou K, Resch B, Sagl G, Petutschnig A, Werner C, Niederseer D, Liedlgruber M, Wilhelm F, Osborne T and Pykett J (2019). Detecting Moments of Stress from Measurements of Wearable Physiological Sensors. Sensors (Basel) 19:(17).

Laurie SS, Lee SMC, Macias BR, Patel N, Stern C, Young M and Stenger MB (2019). Optic Disc Edema and Choroidal Engorgement in Astronauts During Spaceflight and Individuals Exposed to Bed Rest. JAMA Ophthalmol.

Lee AG (2018). Optical Coherence Tomographic Analysis of the Optic Nerve Head and Surrounding Structures in Space Flight- 
Associated Neuro-ocular Syndrome. JAMA Ophthalmol 136:(2): 200-201.

Lee AG, Mader TH, Gibson CR, Brunstetter TJ and Tarver WJ (2018). Space flight-associated neuro-ocular syndrome (SANS). Eye (Lond) 32:(7): 1164-1167.

Lee AG, Mader TH, Gibson CR and Tarver W (2017). Space FlightAssociated Neuro-ocular Syndrome. JAMA Ophthalmol 135:(9): 992-994.

Lee AG, Mader TH, Gibson CR, Tarver W, Rabiei P, Riascos RF, Galdamez LA and Brunstetter T (2020). Spaceflight associated neuroocular syndrome (SANS) and the neuro-ophthalmologic effects of microgravity: a review and an update. NPJ Microgravity 6: 7 .

Lee AG, Tarver WJ, Mader TH, Gibson CR, Hart SF and Otto CA (2016). Neuro-Ophthalmology of Space Flight. J Neuroophthalmol 36:(1): 85-91.

Mader TH, Gibson CR, Miller NR, Subramanian PS, Patel NB and Lee AG (2019). An overview of spaceflight-associated neuro-ocular syndrome (SANS). Neurol India 67:(Supplement): S206-S211.

Mader TH, Gibson CR, Pass AF, Kramer LA, Lee AG, Fogarty J, Tarver WJ, Dervay JP, Hamilton DR, Sargsyan A, Phillips JL, Tran D, Lipsky W, Choi J, Stern C, Kuyumjian R and Polk JD (2011). Optic disc edema, globe flattening, choroidal folds, and hyperopic shifts observed in astronauts after long-duration space flight. Ophthalmology 118:(10): 2058-2069.

Mader TH, Gibson CR, Pass AF, Lee AG, Killer HE, Hansen HC, Dervay JP, Barratt MR, Tarver WJ, Sargsyan AE, Kramer LA, Riascos R, Bedi DG and Pettit DR (2013). Optic disc edema in an astronaut after repeat long-duration space flight. J Neuroophthalmol 33:(3): 249-255.

Mao XW, Boerma M, Rodriguez D, Campbell-Beachler M, Jones T, Stanbouly S, Sridharan V, Nishiyama NC, Wroe A and Nelson GA (2019). Combined Effects of Low-Dose Proton Radiation and Simulated Microgravity on the Mouse Retina and the Hematopoietic System. Radiat Res 192:(3): 241-250.

Mao XW, Nishiyama NC, Byrum SD, Stanbouly S, Jones T, Drew A, Sridharan V, Boerma M, Tackett AJ, Zawieja D, Willey JS, Delp M and Pecaut MJ (2019). Characterization of mouse ocular response to a 35-day spaceflight mission: Evidence of blood-retinal barrier disruption and ocular adaptations. Sci Rep 9:(1): 8215.

Moran EP, Wang Z, Chen J, Sapieha P, Smith LE and Ma JX (2016). Neurovascular cross talk in diabetic retinopathy: Pathophysiological roles and therapeutic implications. Am J Physiol Heart Circ Physiol 311:(3): H738-749.

Morey-Holton E, Globus RK, Kaplansky A and Durnova G (2005). The hindlimb unloading rat model: literature overview, technique update and comparison with space flight data. Adv Space Biol Med 10: 7-40.

Morey-Holton ER and Globus RK (2002). Hindlimb unloading rodent model: technical aspects. J Appl Physiol (1985) 92:(4): 1367-1377.

Musacchia XJ and Fagette S (1997). Weightlessness simulations for cardiovascular and muscle systems: validity of rat models. J Gravit Physiol 4:(3): 49-59.
Nadal-Nicolas FM, Vidal-Sanz M and Agudo-Barriuso M (2018). The aging rat retina: from function to anatomy. Neurobiol Aging 61: 146-168.

Nuzzi R, Scalabrin S, Becco A and Panzica G (2018). Gonadal Hormones and Retinal Disorders: A Review. Front Endocrinol (Lausanne) 9: 66.

Overbey EG, da Silveira WA, Stanbouly S, Nishiyama NC, RoqueTorres GD, Pecaut MJ, Zawieja DC, Wang C, Willey JS, Delp MD, Hardiman G and Mao XW (2019). Spaceflight influences gene expression, photoreceptor integrity, and oxidative stress-related damage in the murine retina. Sci Rep 9:(1): 13304.

Pan FY, Zhang SZ, Xu N, Meng FL, Zhang HX, Xue B, Han X and Li CJ (2010). Beta-catenin signaling involves HGF-enhanced HepG2 scattering through activating MMP-7 transcription. Histochem Cell Biol 134:(3): 285-295.

Ravi D, Beheshti A, Abermil N, Passero F, Sharma J, Coyle M, Kritharis A, Kandela I, Hlatky L, Sitkovsky MV, Mazar A, Gartenhaus RB and Evens AM (2016). Proteasomal Inhibition by Ixazomib Induces CHK1 and MYC-Dependent Cell Death in T-cell and Hodgkin Lymphoma. Cancer Res 76:(11): 3319-3331.

Roque-Torres GD, Nishiyama NC, Stanbouly S and Mao XW (2020). Assessment of Global Ocular Structure Following Spaceflight Using a Micro-Computed Tomography (Micro-CT) Imaging Method. J Vis $\operatorname{Exp}(164)$.

Rubsam A, Parikh S and Fort PE (2018). Role of Inflammation in Diabetic Retinopathy. Int J Mol Sci 19:(4).

Sadaka A and Giuliari GP (2012). Proliferative vitreoretinopathy: current and emerging treatments. Clin Ophthalmol 6: 1325-1333.

Saeed AI, Bhagabati NK, Braisted JC, Liang W, Sharov V, Howe EA, Li J, Thiagarajan M, White JA and Quackenbush J (2006). TM4 microarray software suite. Methods Enzymol 411: 134-193.

Selvam S, Kumar T and Fruttiger M (2018). Retinal vasculature development in health and disease. Prog Retin Eye Res 63: 1-19.

Stenger MB, Laurie SS, Sadda SR, Sadun AA, Macias BR and Huang AS (2019). Focus on the Optic Nerve Head in SpaceflightAssociated Neuro-ocular Syndrome. Ophthalmology 126:(12): 1604-1606.

Subramanian A, Tamayo P, Mootha VK, Mukherjee S, Ebert BL, Gillette MA, Paulovich A, Pomeroy SL, Golub TR, Lander ES and Mesirov JP (2005). Gene set enrichment analysis: a knowledgebased approach for interpreting genome-wide expression profiles. Proc Natl Acad Sci U S A 102:(43): 15545-15550.

Tahimic CGT, Paul AM, Schreurs AS, Torres SM, Rubinstein L, Steczina S, Lowe M, Bhattacharya S, Alwood JS, Ronca AE and Globus RK (2019). Influence of Social Isolation During Prolonged Simulated Weightlessness by Hindlimb Unloading. Front Physiol 10: 1147.

Taibbi G, Cromwell RL, Zanello SB, Yarbough PO, Ploutz-Snyder RJ, Godley BF and Vizzeri G (2016). Ocular Outcomes Comparison Between 14- and 70-Day Head-Down-Tilt Bed Rest. Invest Ophthalmol Vis Sci 57:(2): 495-501. 
Tamm ER, Ethier CR, Lasker IloA and Glaucomatous Neurodegeneration P (2017). Biological aspects of axonal damage in glaucoma: A brief review. Exp Eye Res 157: 5-12.

Templeton JP, Wang X, Freeman NE, Ma Z, Lu A, Hejtmancik F and Geisert EE (2013). A crystallin gene network in the mouse retina. Exp Eye Res 116: 129-140.

Theriot CA, Zanello S.B. (2015). Molecular Effects of Spaceflight in the Mouse Eye after Shuttle Mission STS-135. Gravitational Space Research 2:(1): 3-14.

van Albada ME, Bartelds B, Wijnberg H, Mohaupt S, Dickinson MG, Schoemaker RG, Kooi K, Gerbens F and Berger RM (2010). Gene expression profile in flow-associated pulmonary arterial hypertension with neointimal lesions. Am J Physiol Lung Cell Mol Physiol 298:(4): L483-491.

Wojcik P, Kini A, Al Othman B, Galdamez LA and Lee AG (2020). Spaceflight associated neuro-ocular syndrome. Curr Opin Neurol 33:(1): 62-67.

Wostyn P, Mader TH, Gibson CR and Killer HE (2020). The perivascular space of the central retinal artery as a potential major cerebrospinal fluid inflow route: implications for optic disc edema in astronauts. Eye (Lond) 34:(4): 779-780.

Xu J, Burgoyne PS and Arnold AP (2002). Sex differences in sex chromosome gene expression in mouse brain. Hum Mol Genet 11:(12): 1409-1419.

Yasuda M, Tanaka Y, Ryu M, Tsuda S and Nakazawa T (2014). RNA sequence reveals mouse retinal transcriptome changes early after axonal injury. PLoS One 9:(3): e93258.

Zhang LF and Hargens AR (2018). Spaceflight-Induced Intracranial Hypertension and Visual Impairment: Pathophysiology and Countermeasures. Physiol Rev 98:(1): 59-87.

Zwart SR, Gibson CR, Gregory JF, Mader TH, Stover PJ, Zeisel SH and Smith SM (2017). Astronaut ophthalmic syndrome. FASEB J 31:(9): 3746-3756.

Zwart SR, Gibson CR, Mader TH, Ericson K, Ploutz-Snyder R, Heer $M$ and Smith SM (2012). Vision changes after spaceflight are related to alterations in folate- and vitamin B-12-dependent onecarbon metabolism. J Nutr 142:(3): 427-431.

Zwart SR, Gregory JF, Zeisel SH, Gibson CR, Mader TH, Kinchen JM, Ueland PM, Ploutz-Snyder R, Heer MA and Smith SM (2016). Genotype, B-vitamin status, and androgens affect spaceflightinduced ophthalmic changes. FASEB J 30:(1): 141-148.

Zwart SR, Laurie SS, Chen JJ, Macias BR, Lee SMC, Stenger M, Grantham B, Carey K, Young M and Smith SM (2019). Association of Genetics and B Vitamin Status With the Magnitude of Optic Disc Edema During 30-Day Strict Head-Down Tilt Bed Rest. JAMA Ophthalmol. 